\title{
Research on Automatic Control Strategy of Direct Air-cooling System for 660 MW Thermal Power Plants
}

\author{
Gao Song ${ }^{1, a}$, Gao Bo ${ }^{2, b}$, Meng Xiangrong ${ }^{1, a}$ \\ ${ }^{1}$ State Grid Shan Dong Electric Power Research Institute, Jinan 250003, China \\ 2 Shandong Shengli Steel Pipe Co., Ltd. Zibo 255082, China \\ agaosong163mail@163.com, bredblack9@163.com
}

Keywords: Direct Air-cooling System, Automatic control Strategy, 660MW Thermal Power Plants

\begin{abstract}
This research focuses on automatic control strategy of direct air-cooling system for 660 MW thermal power plants. The aim of the controller is to maintain the backpressure in an adjustable range. The speed control policy takes the backpressure to the controllable range by adjusting the running fan speed, and the sequential control policy starts or shutoffs the pre specified fans when the backpressure is out of the normal range. An application of $660 \mathrm{MW}$ power unit shows that the automatic control strategy has important significance for the safe and economic operation of generating units.
\end{abstract}

\section{Introduction}

Backpressure is an important parameter of direct air-cooling system for large-scale thermal power plants, so the automatic control strategy of back pressure is widely studied [1-3]. Taking effective automatic control strategy to set the backpressure into an adjustable range, it has important significance for the safe operation of power unit, and also helps to improve the economic index of the unit operation [4-7].

\section{Direct Air-cooling System}

Ningxia Yuanyang Lake Power Plant has 2 x 660MW supercritical direct air-cooling units. The air-cooling island is provided by SPX Company, and its control scheme is incorporated into the distributed control system (DCS). The DCS is Industrial IT System 800xA by ABB Bailey Beijing engineering company.

The direct air-cooling system of Yuanyang Lake Power Plant is as shown in Fig.1.

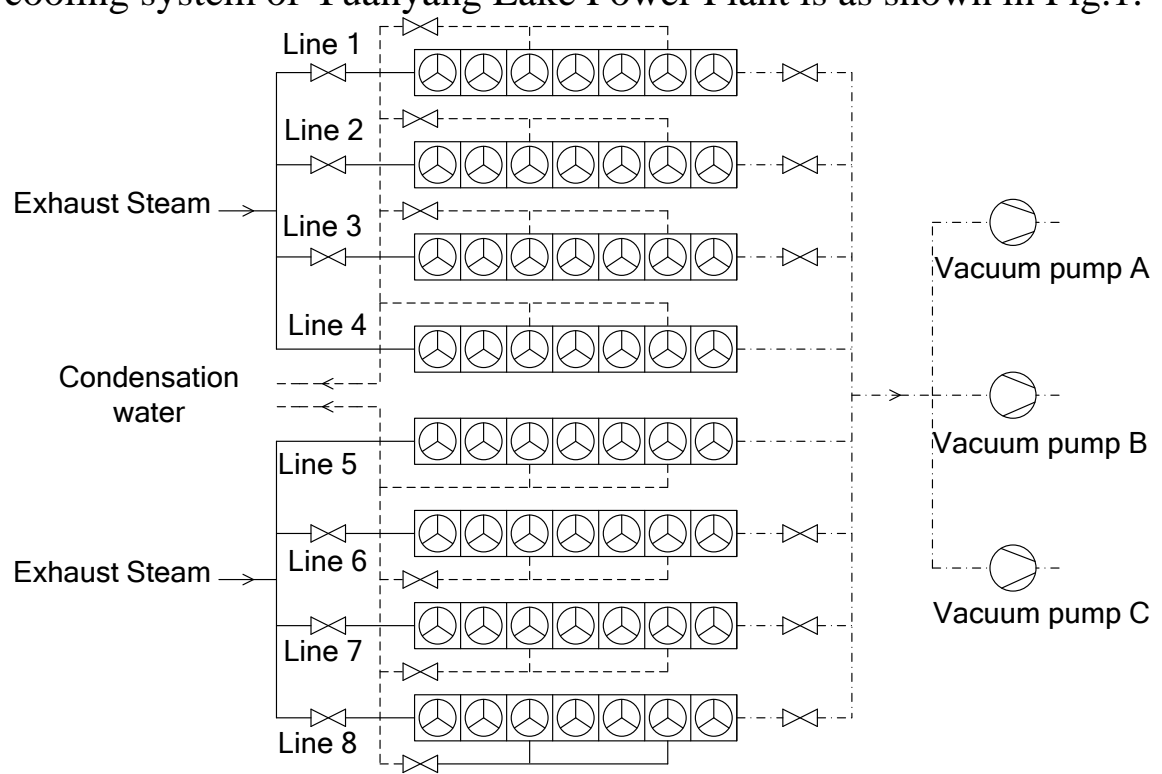

Fig.1 Direct air-cooling system of Yuanyang Lake Power Plant 
The air-cooled steam condenser (ACC) condenses the turbine exhaust steam or the de-superheated steam from the turbine bypass by rejecting the heat to the atmosphere. The condenser consists of 8 "A" roof-type streets of finned tube elements. Each street contains 7 modules ( 5 primary and 2 mixed modules). Each module is composed of 10 bundles of finned tubes. An axial flow fan located underneath the roof forces the cooling air through the fins. The steam flows through two large diameter ducts to the condenser. The duct system branches into 8 risers and steam distribution manifolds running along the top of each street. Steam enters from this distribution manifold into the finned tubes at the top of the primary condensing bundles.

\section{Automatic control strategy of ACC}

The turbine backpressure is measured by the pressure transmitter on the main steam ducts and serves to control fan operation. The aim of automatic control for air-cooling system is to regulate the pressure as the set point value. The control strategy is divided into two parts: fan speed control and sequential control, as shown in Fig.2.

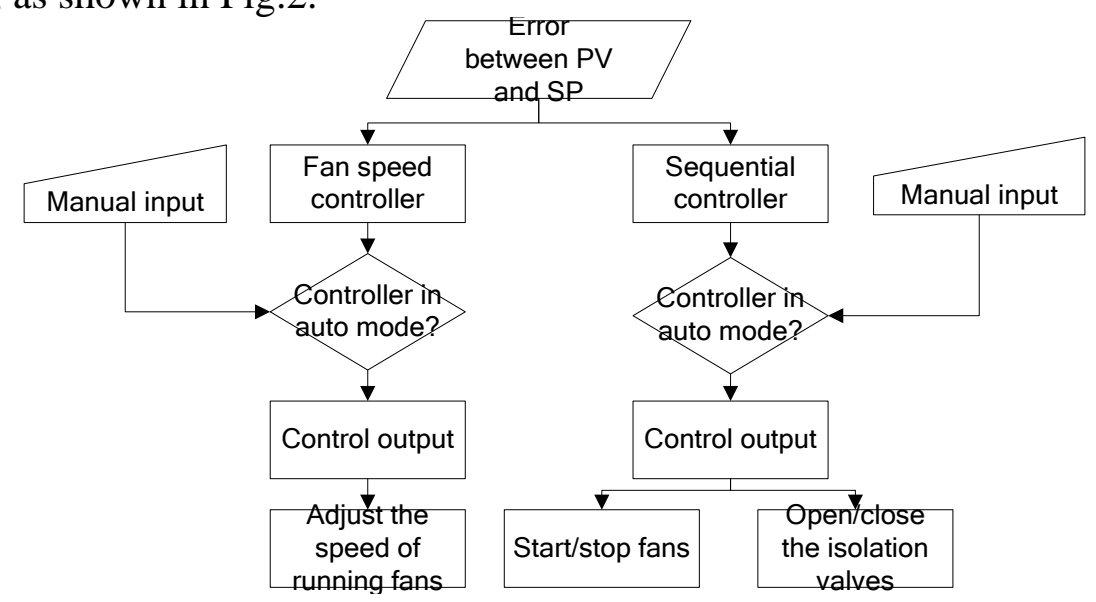

Fig.2 Automatic control strategy of ACC

Fan Speed Control. The back pressure actual value is compared with the set value, and is used for a PID control input. Through the PID controller the speed of all the running fans is adjusted to maintain the backpressure into a suitable range. The fan speed control flow chart is shown in Fig.3.

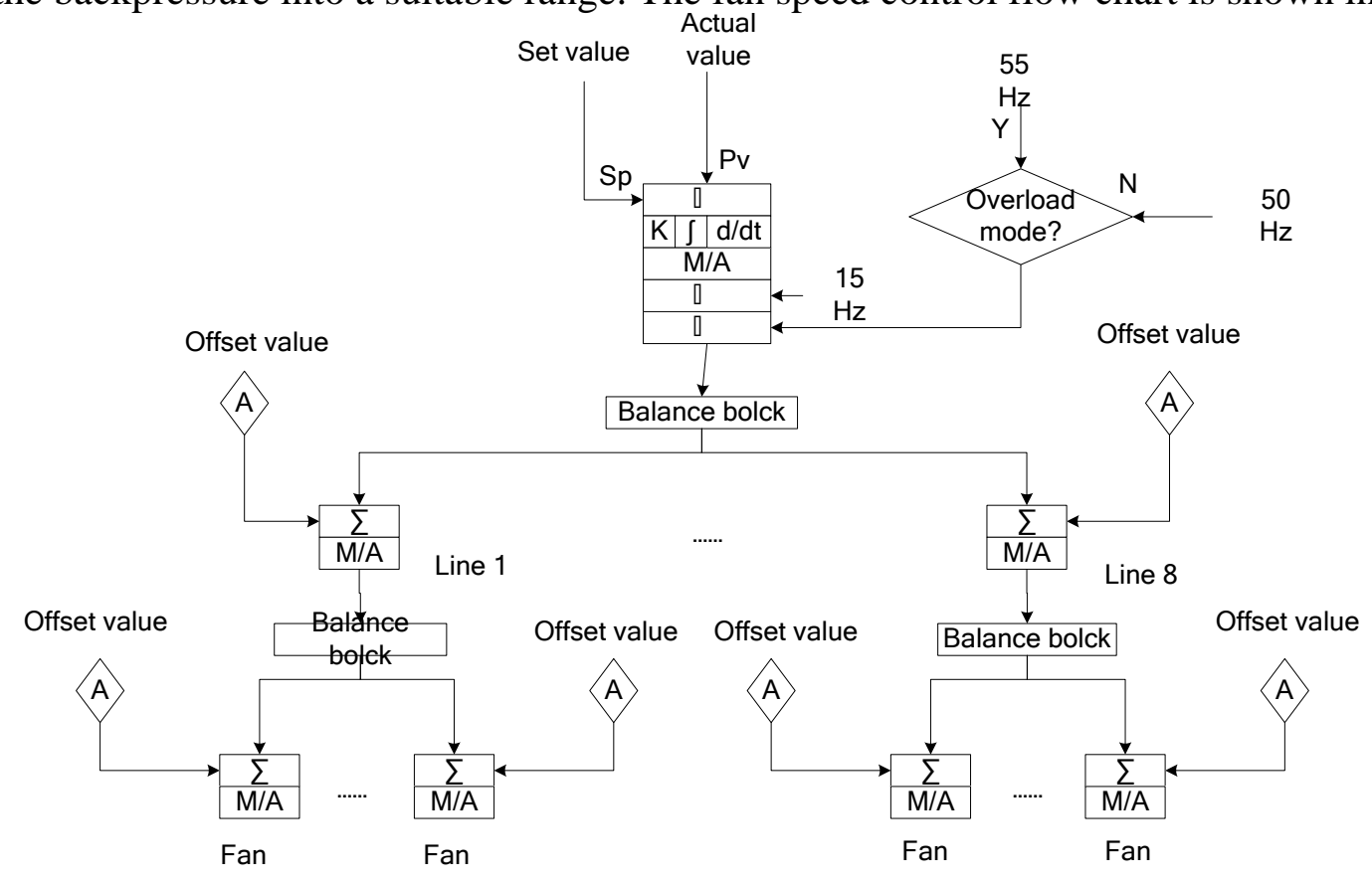

Fig.3 Fan speed control strategy 
To protect the equipment, lower limit of fan start frequency is set to $15 \mathrm{~Hz}$, the upper limit of $50 \mathrm{~Hz}$, only in overload mode the frequency limit of $50 \mathrm{~Hz}$ is up to $55 \mathrm{~Hz}$.

In order to make the automatic operation can be coordinated with manual operation, an offset value of $\mathrm{M} / \mathrm{A}$ block of the fan speed control is settled.

Sequential Control. when the actual backpressure is far off away from the set value, the sequential control will start more fans to maintain the backpressure, otherwise, the sequential control will shutoff fans to avoid too low backpressure.

The sequential control has 16 steps, and each step is mapping to different numbers of running fans. The control steps are S0, S1, ..,S15, with S0 is the initial state, and the last step S15 is overload mode.

The sequential control is shown as Fig.4.
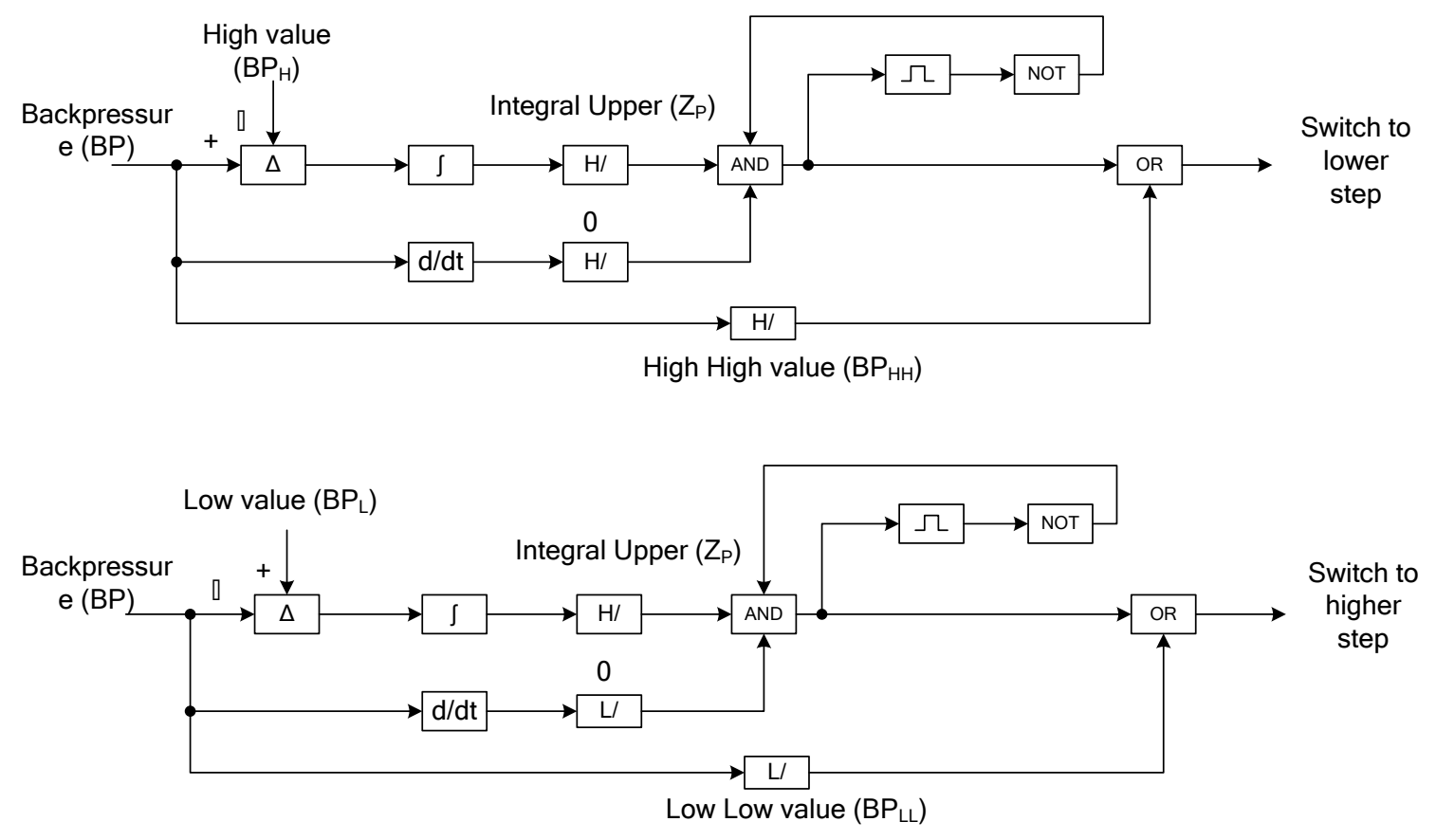

Fig.4 Sequential control strategy

The sequential control will maintain the backpressure in a suitable range, $B P_{L}<B P<B P_{H}$, with the high value $B P_{H}=1.1 B P_{S P}$, and the low value $B P_{L}=0.9 B P_{S P}$. In this range, the controller will keep current step.

When the backpressure is out of the adjusted range, the error between actual value and set value will be calculated by an integrator. If the integral value is greater than the integral upper $Z_{P}$, the controller switches to a higher or lower control step which will start or shutoff the pre specified fans. If the backpressure is back into the adjusted range, the integral value will be reset to zero.

In order to avoid a too frequent step switching, an adjustable dead-time period of at least 1 minute is applicable after a step switching.

\section{Application Result}

The application of automatic control strategy for Ningxia Yuanyang Lake Power Plant 660MW supercritical direct air-cooling units is shown in Fig.5.

When the set point changes, the control output can quickly response and the controller will quickly adjust the fan speed and switch the control step, so the process value can quickly return to the adjustable range of set point and overshot is small. 


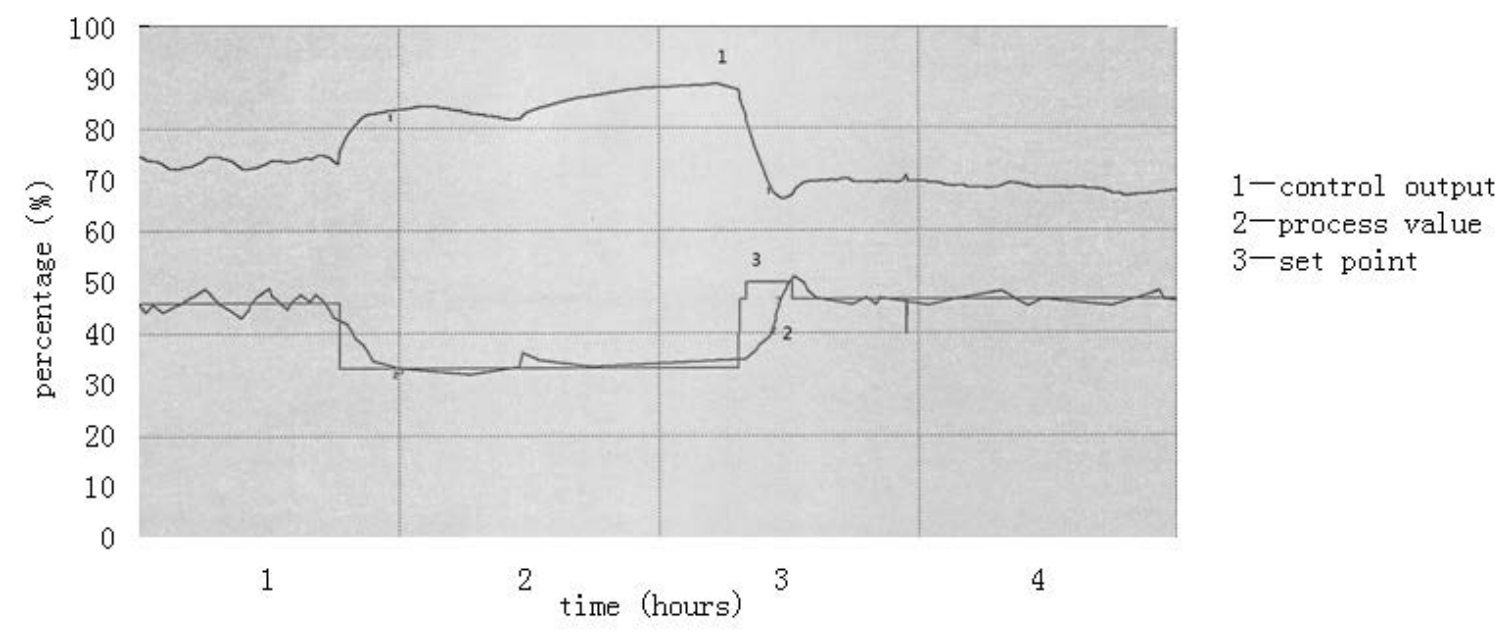

Fig.5 Application result of $660 \mathrm{MW}$ power unit

\section{Conclusion}

Through the application of automatic control strategy of direct air-cooling system for Yuanyang Lake 660MW power units, the backpressure is controlled within a tight band, fast response, and small overshot, therefore it has important significance for the safe and economic operation of generating units. The backpressure is affected by the ambient temperature. The further work is to research the relationship between the backpressure and the ambient temperature, and improve the control strategy with feed forward control of ambient temperature.

\section{References}

[1] Bai Jian-yun, Wang Yingxu. Program control strategies for the direct air-cooling system. Electric Power, Vol. 41, No. 4, 2008.

[2] Zhang Xinhai. Discussion on direct ACC system of 1000MW ultra-supercritical unit. Huadian Technology, Vol. 30, No. 5, 2008.

[3] GUO Yufeng, QIN Hua, YU Daren, ZHAO Xiaomin. Influences of Cooling Air Face Velocity and Temperature on Dynamic Characteristics of Direct Air-cooled System. Proceeding of the CSEE, 28(29): 22-27, 2008.

[4] Bai Jianyun, Shao Jiaxiao, Hou Pengfei. 600MW Direct Air Cooling Unit Back-pressure Control Strategy Design and Optimization. Power System Engineering, Vol. 27, No. 2, 2011.

[5] ZHANG XiaoLu,WANG JianBeng,XUN Rui. Optimal Design Method of Direct Air-cooling System for Thermal Power Plants. Proceeding of the CSEE, 31(11): 1-5, 2011.

[6] He Jun, Liu Baoling, Yan Bo. Optimization of backpressure control strategy for a $600 \mathrm{MW}$ supercritical direct air-cooled unit. Thermal Power Generation, Vol. 42, No. 7, 2013.

[7] Hong Bin Zhao et al. 600 MW Direct Air-Cooling Unit Back-Pressure Optimization and Theoretical Analysis. Advanced Materials Research, 875-877, 1739, 2014. 